\title{
Some Facts about Trigonometry and Euclidean Geometry
}

\author{
Roland Coghetto \\ Rue de la Brasserie 5 \\ 7100 La Louvière, Belgium
}

\begin{abstract}
Summary. We calculate the values of the trigonometric functions for angles: $\frac{\pi}{3}$ and $\frac{\pi}{6}$, by [16]. After defining some trigonometric identities, we demonstrate conventional trigonometric formulas in the triangle, and the geometric property, by [14], of the triangle inscribed in a semicircle, by the proposition 3.31 in 15. Then we define the diameter of the circumscribed circle of a triangle using the definition of the area of a triangle and prove some identities of a triangle [9]. We conclude by indicating that the diameter of a circle is twice the length of the radius.
\end{abstract}

MSC: 51M04 03B35

Keywords: Euclidean geometry; trigonometry; circumcircle; right-angled

MML identifier: EUCLID10, version: 8.1.03 5.26.1224

The notation and terminology used in this paper have been introduced in the following articles: [1], 10], [11, [19], 25], [3], 12], [5], 21], 22, 28], 66, 7], 24], [29], 23], [18], [26], 27], [13], and [8].

1. Values of the Trigonometric Functions for Angles: $\frac{\pi}{3}$ And $\frac{\pi}{6}$

Let us consider a real number $a$. Now we state the propositions:

(1) $\sin (\pi-a)=\sin a$.

(2) $\cos (\pi-a)=-\cos a$.

(3) $\sin (2 \cdot \pi-a)=-\sin a$.

(4) $\cos (2 \cdot \pi-a)=\cos a$.

(5) $\sin (-2 \cdot \pi+a)=\sin a$. 
(6) $\cos (-2 \cdot \pi+a)=\cos a$.

(7) $\sin \left(\frac{3 \cdot \pi}{2}+a\right)=-\cos a$.

(8) $\cos \left(\frac{3 \cdot \pi}{2}+a\right)=\sin a$.

(9) $\sin \left(\frac{3 \cdot \pi}{2}+a\right)=-\sin \left(\frac{\pi}{2}-a\right)$. The theorem is a consequence of $(7)$.

(10) $\cos \left(\frac{3 \cdot \pi}{2}+a\right)=\cos \left(\frac{\pi}{2}-a\right)$. The theorem is a consequence of (8).

(11) $\sin \left(\frac{2 \cdot \pi}{3}-a\right)=\sin \left(\frac{\pi}{3}+a\right)$.

(12) $\cos \left(\frac{2 \cdot \pi}{3}-a\right)=-\cos \left(\frac{\pi}{3}+a\right)$.

(13) $\sin \left(\frac{2 \cdot \pi}{3}+a\right)=\sin \left(\frac{\pi}{3}-a\right)$.

Now we state the propositions:

(14) $\cos \frac{\pi}{3}=\frac{1}{2}$.

(15) $\sin \frac{\pi}{3}=\frac{\sqrt{3}}{2}$.

ProOF: $\sin \frac{\pi}{3} \geqslant 0$ by [20, (5)], [29, (79), (81)].

(16) $\operatorname{tg} \frac{\pi}{3}=\sqrt{3}$. The theorem is a consequence of (14) and (15).

(17) $\sin \frac{\pi}{6}=\frac{1}{2}$. The theorem is a consequence of (14).

(18) $\cos \frac{\pi}{6}=\frac{\sqrt{3}}{2}$. The theorem is a consequence of (15).

(19) $\operatorname{tg} \frac{\pi}{6}=\frac{\sqrt{3}}{3}$. The theorem is a consequence of (17) and (18).

(20) (i) $\sin \left(-\frac{\pi}{6}\right)=-\frac{1}{2}$, and

(ii) $\cos \left(-\frac{\pi}{6}\right)=\frac{\sqrt{3}}{2}$, and

(iii) $\operatorname{tg}\left(-\frac{\pi}{6}\right)=-\frac{\sqrt{3}}{3}$, and

(iv) $\sin \left(-\frac{\pi}{3}\right)=-\frac{\sqrt{3}}{2}$, and

(v) $\cos \left(-\frac{\pi}{3}\right)=\frac{1}{2}$, and

(vi) $\operatorname{tg}\left(-\frac{\pi}{3}\right)=-\sqrt{3}$.

(21) (i) $\arcsin \frac{1}{2}=\frac{\pi}{6}$, and

(ii) $\arcsin \frac{\sqrt{3}}{2}=\frac{\pi}{3}$.

The theorem is a consequence of (15) and (17).

(22) $\sin \frac{2 \cdot \pi}{3}=\frac{\sqrt{3}}{2}$. The theorem is a consequence of (11) and (15).

(23) $\cos \frac{2 \cdot \pi}{3}=-\frac{1}{2}$. The theorem is a consequence of (12) and (14).

\section{Some Trigonometric Identities}

Now we state the proposition:

(24) Let us consider a real number $x$. Then $(\sin (-x))^{2}=(\sin x)^{2}$.

Let us consider real numbers $x, y, z$. Now we state the propositions:

(25) If $x+y+z=\pi$, then $(\sin x)^{2}+(\sin y)^{2}-2 \cdot \sin x \cdot \sin y \cdot \cos z=(\sin z)^{2}$. 
(26) If $x-y+z=\pi$, then $(\sin x)^{2}+(\sin y)^{2}+2 \cdot \sin x \cdot \sin y \cdot \cos z=(\sin z)^{2}$. The theorem is a consequence of (24) and (25).

(27) Suppose $x-(-2 \cdot \pi+y)+z=\pi$. Then $(\sin x)^{2}+(\sin y)^{2}+2 \cdot \sin x \cdot \sin y$. $\cos z=(\sin z)^{2}$. The theorem is a consequence of (24), (5), and (25).

(28) If $\pi-x-(\pi-y)+z=\pi$, then $(\sin x)^{2}+(\sin y)^{2}+2 \cdot \sin x \cdot \sin y \cdot \cos z=$ $(\sin z)^{2}$. The theorem is a consequence of $(24),(1)$, and $(25)$.

Now we state the proposition:

(29) Let us consider a real number $a$. Then $\sin (3 \cdot a)=4 \cdot \sin a \cdot \sin \left(\frac{\pi}{3}+a\right)$. $\sin \left(\frac{\pi}{3}-a\right)$. The theorem is a consequence of $(15)$.

\section{Trigonometric Functions and Right Triangle}

Let us consider points $A, B, C$ of $\mathcal{E}_{\mathrm{T}}^{2}$.

Let us assume that $A, B, C$ form a triangle. Now we state the propositions:

(30) (i) $\measuredangle(A, B, C)$ is not zero, and

(ii) $\measuredangle(B, C, A)$ is not zero, and

(iii) $\measuredangle(C, A, B)$ is not zero, and

(iv) $\measuredangle(A, C, B)$ is not zero, and

(v) $\measuredangle(C, B, A)$ is not zero, and

(vi) $\measuredangle(B, A, C)$ is not zero.

(i) $\measuredangle(A, B, C)=2 \cdot \pi-\measuredangle(C, B, A)$, and

(ii) $\measuredangle(B, C, A)=2 \cdot \pi-\measuredangle(A, C, B)$, and

(iii) $\measuredangle(C, A, B)=2 \cdot \pi-\measuredangle(B, A, C)$, and

(iv) $\measuredangle(B, A, C)=2 \cdot \pi-\measuredangle(C, A, B)$, and

(v) $\measuredangle(A, C, B)=2 \cdot \pi-\measuredangle(B, C, A)$, and

(vi) $\measuredangle(C, B, A)=2 \cdot \pi-\measuredangle(A, B, C)$.

Now we state the proposition:

(32) Suppose $A, B, C$ form a triangle and $|(B-A, C-A)|=0$. Then

(i) $|C-B| \cdot \sin \measuredangle(C, B, A)=|A-C|$, or

(ii) $|C-B| \cdot(-\sin \measuredangle(C, B, A))=|A-C|$.

Let us assume that $A, B, C$ form a triangle and $\measuredangle(B, A, C)=\frac{\pi}{2}$. Now we state the propositions:

(33) $\measuredangle(C, B, A)+\measuredangle(A, C, B)=\frac{\pi}{2}$.

(34) (i) $|C-B| \cdot \sin \measuredangle(C, B, A)=|A-C|$, and 
(ii) $|C-B| \cdot \sin \measuredangle(A, C, B)=|A-B|$, and

(iii) $|C-B| \cdot \cos \measuredangle(C, B, A)=|A-B|$, and

(iv) $|C-B| \cdot \cos \measuredangle(A, C, B)=|A-C|$.

(i) $\operatorname{tg} \measuredangle(A, C, B)=\frac{|A-B|}{|A-C|}$, and

(ii) $\operatorname{tg} \measuredangle(C, B, A)=\frac{|A-C|}{|A-B|}$.

The theorem is a consequence of (34).

\section{Triangle Inscribed in a Semicircle is a Right Triangle}

Let $a, b$ be real numbers and $r$ be a negative real number. Let us note that $\operatorname{circle}(a, b, r)$ is empty.

Now we state the proposition:

(36) Let us consider real numbers $a, b$. Then $\operatorname{circle}(a, b, 0)=\{[a, b]\}$.

Let $a, b$ be real numbers. One can verify that $\operatorname{circle}(a, b, 0)$ is trivial.

Now we state the propositions:

(37) Let us consider points $A, B, C$ of $\mathcal{E}_{\mathrm{T}}^{2}$, and real numbers $a, b, r$. Suppose $A, B, C$ form a triangle and $A, B \in \operatorname{circle}(a, b, r)$. Then $r$ is positive. The theorem is a consequence of (36).

(38) Let us consider a point $A$ of $\mathcal{E}_{\mathrm{T}}^{2}$, real numbers $a, b$, and a positive real number $r$. If $A \in \operatorname{circle}(a, b, r)$, then $A \neq[a, b]$.

(39) Let us consider points $A, B, C$ of $\mathcal{E}_{\mathrm{T}}^{2}$, and real numbers $a, b, r$. Suppose $A, B, C$ form a triangle and $\measuredangle(C, B, A), \measuredangle(B, A, C) \in] 0, \pi[$ and $A, B$, $C \in \operatorname{circle}(a, b, r)$ and $[a, b] \in \mathcal{L}(A, C)$. Then $\measuredangle(C, B, A)=\frac{\pi}{2}$.

Proof: Set $O=[a, b]$. Consider $J_{1}$ being a point of $\mathcal{E}_{\mathrm{T}}^{2}$ such that $A=J_{1}$ and $\left|J_{1}-[a, b]\right|=r$. Consider $J_{2}$ being a point of $\mathcal{E}_{\mathrm{T}}^{2}$ such that $B=J_{2}$ and $\left|J_{2}-[a, b]\right|=r$. Consider $J_{3}$ being a point of $\mathcal{E}_{\mathrm{T}}^{2}$ such that $C=J_{3}$ and $\mid J_{3}-$ $[a, b] \mid=r . r$ is positive. $O \neq A$ and $O \neq C . \measuredangle(C, B, O)<\pi$ by [25, (16), (9)], [19, (47)]. $A, O, B$ form a triangle and $C, O, B$ form a triangle by (37), (38), [6. (72), (75)]. $\measuredangle(C, B, O)+\measuredangle(O, C, B)+\measuredangle(O, B, A)+\measuredangle(B, A, O)=\pi$ or $\measuredangle(C, B, O)+\measuredangle(O, C, B)+\measuredangle(O, B, A)+\measuredangle(B, A, O)=-\pi$ by [25, (13)], [19, (47)]. $\measuredangle(O, C, B)=\measuredangle(C, B, O)$ and $\measuredangle(B, A, O)=\measuredangle(O, B, A)$.

(40) Let us consider points $A, B, C$ of $\mathcal{E}_{\mathrm{T}}^{2}$, and a positive real number $r$. Suppose $\measuredangle(A, B, C)$ is not zero. Then $\sin (r \cdot \measuredangle(C, B, A))=\sin (r \cdot 2 \cdot \pi) \cdot$ $\cos (r \cdot \measuredangle(A, B, C))-\cos (r \cdot 2 \cdot \pi) \cdot \sin (r \cdot \measuredangle(A, B, C))$.

(41) Let us consider points $A, B, C$ of $\mathcal{E}_{\mathrm{T}}^{2}$. Suppose $\measuredangle(A, B, C)$ is not zero. Then $\sin \frac{\measuredangle(C, B, A)}{3}=\frac{\sqrt{3}}{2} \cdot \cos \frac{\measuredangle(A, B, C)}{3}+\frac{1}{2} \cdot \sin \frac{\measuredangle(A, B, C)}{3}$. The theorem is a consequence of (40), (22), and (23). 


\section{Diameter of the Circumcircle of a Triangle}

Let us consider points $A, B, C$ of $\mathcal{E}_{\mathrm{T}}^{2}$. Now we state the propositions:

(42) (i) area of $\triangle(A, B, C)=$ area of $\triangle(B, C, A)$, and

(ii) area of $\triangle(A, B, C)=$ area of $\triangle(C, A, B)$.

(43) area of $\triangle(A, B, C)=-($ area of $\triangle(B, A, C)$ ).

Let $A, B, C$ be points of $\mathcal{E}_{\mathrm{T}}^{2}$. The functor $\varnothing_{\curvearrowright}(A, B, C)$ yielding a real number is defined by the term

(Def. 1) $\frac{\frac{|A-B| \cdot|B-C| \cdot|C-A|}{2}}{\text { area of } \Delta(A, B, C)}$.

Let us consider points $A, B, C$ of $\mathcal{E}_{\mathrm{T}}^{2}$.

Let us assume that $A, B, C$ form a triangle. Now we state the propositions:

(44) $\varnothing_{0}(A, B, C)=\frac{|C-A|}{\sin \measuredangle(C, B, A)}$.

(45) $\varnothing_{0}(A, B, C)=-\frac{|C-A|}{\sin \measuredangle(A, B, C)}$. The theorem is a consequence of (44).

Now we state the proposition:

(46) $\varnothing_{0}(A, B, C)=\varnothing_{0}(B, C, A)$.

Let us assume that $A, B, C$ form a triangle. Now we state the propositions:

(47) $\varnothing_{0}(A, B, C)=-\varnothing_{\rho}(B, A, C)$. The theorem is a consequence of (43).

(48) $\varnothing_{\supset}(A, B, C)=-\varnothing_{\supset}(A, C, B)$. The theorem is a consequence of (42) and (47).

(49) $\varnothing_{0}(A, B, C)=-\varnothing_{\supset}(C, B, A)$. The theorem is a consequence of (48) and (42).

\section{Some Identities of a Triangle}

Let us consider points $A, B, C$ of $\mathcal{E}_{\mathrm{T}}^{2}$.

Let us assume that $A, B, C$ form a triangle. Now we state the propositions:

(i) $|A-B|=\varnothing_{\supset}(A, B, C) \cdot \sin \measuredangle(A, C, B)$, and

(ii) $|B-C|=\varnothing_{0}(A, B, C) \cdot \sin \measuredangle(B, A, C)$, and

(iii) $|C-A|=\varnothing_{0}(A, B, C) \cdot \sin \measuredangle(C, B, A)$.

The theorem is a consequence of (42).

(51) $|A-B|=\varnothing_{0}(A, B, C) \cdot 4 \cdot \sin \frac{\measuredangle(A, C, B)}{3} \cdot \sin \left(\frac{\pi}{3}+\frac{\measuredangle(A, C, B)}{3}\right) \cdot \sin \left(\frac{\pi}{3}-\frac{\measuredangle(A, C, B)}{3}\right)$.

The theorem is a consequence of (29).

Let us consider points $A, B, C, P$ of $\mathcal{E}_{\mathrm{T}}^{2}$. Now we state the propositions:

(52) Suppose $A, B, P$ are mutually different and $\measuredangle(P, B, A)=\frac{\measuredangle(C, B, A)}{3}$ and $\measuredangle(B, A, P)=\frac{\measuredangle(B, A, C)}{3}$ and $\measuredangle(A, P, B)<\pi$. Then $|A-P| \cdot \sin (\pi-$ $\left.\left(\frac{\measuredangle(C, B, A)}{3}+\frac{\measuredangle(B, A, C)}{3}\right)\right)=|A-B| \cdot \sin \frac{\measuredangle(C, B, A)}{3}$. 
(53) Suppose $A, B, P$ are mutually different and $\measuredangle(P, B, A)=\frac{\measuredangle(C, B, A)}{3}$ and $\measuredangle(B, A, P)=\frac{\measuredangle(B, A, C)}{3}$ and $\measuredangle(A, P, B)<\pi$ and $\frac{\measuredangle(C, B, A)}{3}+\frac{\measuredangle(B, A, C)}{3}+$ $\frac{\measuredangle(A, C, B)}{3}=\frac{\pi}{3}$. Then $|A-P| \cdot \sin \left(\frac{2 \cdot \pi}{3}+\frac{\measuredangle(A, C, B)}{3}\right)=|A-B| \cdot \sin \frac{\measuredangle(C, B, A)}{3}$.

Now we state the proposition:

(54) Let us consider points $A, B, C$ of $\mathcal{E}_{\text {T. }}^{2}$ Suppose $A, B, C$ form a triangle and $\measuredangle(C, A, B)<\pi$. Then

(i) $\measuredangle(C, B, A)+\measuredangle(B, A, C)+\measuredangle(A, C, B)=5 \cdot \pi$, and

(ii) $\measuredangle(C, A, B)+\measuredangle(A, B, C)+\measuredangle(B, C, A)=\pi$.

Let us consider points $A, B, C, P$ of $\mathcal{E}_{\mathrm{T}}^{2}$. Now we state the propositions:

(55) Suppose $A, B, C$ form a triangle and $\measuredangle(C, B, A)<\pi$ and $A, B, P$ are mutually different and $\measuredangle(P, B, A)=\frac{\measuredangle(C, B, A)}{3}$ and $\measuredangle(B, A, P)=\frac{\measuredangle(B, A, C)}{3}$ and $\measuredangle(A, P, B)<\pi$. Then $|A-P| \cdot \sin \left(\frac{\pi}{3}-\frac{\measuredangle(A, C, B)}{3}\right)=|A-B| \cdot \sin \frac{\measuredangle(C, B, A)}{3}$. The theorem is a consequence of (1).

(56) Suppose $A, B, C$ form a triangle and $A, B, P$ form a triangle and $\measuredangle(C, B, A)<\pi$ and $\measuredangle(A, P, B)<\pi$ and $\measuredangle(P, B, A)=\frac{\measuredangle(C, B, A)}{3}$ and $\measuredangle(B, A, P)=\frac{\measuredangle(B, A, C)}{3}$ and $\sin \left(\frac{\pi}{3}-\frac{\measuredangle(A, C, B)}{3}\right) \neq 0$. Then $|A-P|=$ $-\varnothing_{\supset}(C, B, A) \cdot 4 \cdot \sin \frac{\measuredangle(A, C, B)}{3} \cdot \sin \left(\frac{\pi}{3}+\frac{\measuredangle(A, C, B)}{3}\right) \cdot \sin \frac{\measuredangle(C, B, A)}{3}$. The theorem is a consequence of (53), (29), (50), (13), and (49).

\section{Diameter of a Circle}

Now we state the propositions:

(57) Let us consider points $A, B, C$ of $\mathcal{E}_{\mathrm{T}}^{2}$. Suppose $A, B, C$ are mutually different and $C \in \mathcal{L}(A, B)$. Then $|A-B|=|A-C|+|C-B|$.

(58) Let us consider points $A, B$ of $\mathcal{E}_{\mathrm{T}}^{2}$, real numbers $a, b$, and a positive real number $r$. Suppose $A, B,[a, b]$ are mutually different and $A, B \in$ $\operatorname{circle}(a, b, r)$ and $[a, b] \in \mathcal{L}(A, B)$. Then $|A-B|=2 \cdot r$. The theorem is a consequence of (57).

(59) Let us consider real numbers $a, b$, a positive real number $r$, and a subset $C$ of $\mathcal{E}^{2}$. If $C=\operatorname{circle}(a, b, r)$, then $\varnothing C=2 \cdot r$.

Proof: For every points $x, y$ of $\mathcal{E}^{2}$ such that $x, y \in C$ holds $\rho(x, y) \leqslant 2 \cdot r$ by [11, (22), (67)], [17, (4)], [22, (5)]. For every real number $s$ such that for every points $x, y$ of $\mathcal{E}^{2}$ such that $x, y \in C$ holds $\rho(x, y) \leqslant s$ holds $2 \cdot r \leqslant s$ by [11, (62)], [4, (12)], [19, (24)], [26, (22)]. 


\section{REFERENCES}

[1] Grzegorz Bancerek. Cardinal numbers Formalized Mathematics, 1(2):377-382, 1990.

[2] Grzegorz Bancerek. The ordinal numbers. Formalized Mathematics, 1(1):91-96, 1990.

[3] Grzegorz Bancerek and Krzysztof Hryniewiecki. Segments of natural numbers and finite sequences. Formalized Mathematics, 1(1):107-114, 1990.

[4] Czesław Byliński. The complex numbers. Formalized Mathematics, 1(3):507-513, 1990.

[5] Czesław Byliński. Functions and their basic properties Formalized Mathematics, 1(1): 55-65, 1990.

[6] Czesław Byliński. Introduction to real linear topological spaces. Formalized Mathematics, 13(1):99-107, 2005.

[7] Czesław Byliński. The sum and product of finite sequences of real numbers Formalized Mathematics, 1(4):661-668, 1990.

[8] Czesław Byliński. Some basic properties of sets Formalized Mathematics, 1(1):47-53, 1990.

[9] H.S.M. Coxeter and S.L. Greitzer. Geometry Revisited. The Mathematical Association of America (Inc.), 1967.

[10] Agata Darmochwał. Compact spaces Formalized Mathematics, 1(2):383-386, 1990.

[11] Agata Darmochwał. The Euclidean space. Formalized Mathematics, 2(4):599-603, 1991.

[12] Agata Darmochwał. Finite sets Formalized Mathematics, 1(1):165-167, 1990.

[13] Alicia de la Cruz. Totally bounded metric spaces Formalized Mathematics, 2(4):559-562, 1991.

[14] Nikolai Vladimirovich Efimov. Géométrie supérieure. Mir, 1981.

[15] Richard Fitzpatrick. Euclid's Elements. Lulu.com, 2007.

[16] Robin Hartshorne. Geometry: Euclid and beyond. Springer, 2000.

[17] Stanisława Kanas, Adam Lecko, and Mariusz Startek. Metric spaces Formalized Mathematics, 1(3):607-610, 1990.

[18] Artur Korniłowicz and Yasunari Shidama. Inverse trigonometric functions arcsin and arccos. Formalized Mathematics, 13(1):73-79, 2005.

[19] Akihiro Kubo and Yatsuka Nakamura. Angle and triangle in Euclidean topological space Formalized Mathematics, 11(3):281-287, 2003.

[20] Robert Milewski. Trigonometric form of complex numbers Formalized Mathematics, 9 (3):455-460, 2001.

[21] Yatsuka Nakamura. General Fashoda meet theorem for unit circle and square Formalized Mathematics, 11(3):213-224, 2003.

[22] Yatsuka Nakamura and Czesław Byliński. Extremal properties of vertices on special polygons. Part I. Formalized Mathematics, 5(1):97-102, 1996.

[23] Chanapat Pacharapokin, Kanchun, and Hiroshi Yamazaki. Formulas and identities of trigonometric functions. Formalized Mathematics, 12(2):139-141, 2004.

[24] Beata Padlewska. Families of sets Formalized Mathematics, 1(1):147-152, 1990.

[25] Marco Riccardi. Heron's formula and Ptolemy's theorem. Formalized Mathematics, 16 (2):97-101, 2008. doi 10.2478/v10037-008-0014-2

[26] Andrzej Trybulec and Czesław Byliński. Some properties of real numbers Formalized Mathematics, 1(3):445-449, 1990.

[27] Zinaida Trybulec. Properties of subsets Formalized Mathematics, 1(1):67-71, 1990.

[28] Edmund Woronowicz. Relations and their basic properties Formalized Mathematics, 1 (1):73-83, 1990.

[29] Yuguang Yang and Yasunari Shidama. Trigonometric functions and existence of circle ratio Formalized Mathematics, 7(2):255-263, 1998. 